\title{
Power Optimization of Tilted Tomlinson-Harashima Precoder in MIMO Channels with Imperfect Channel State Information
}

\author{
Hossein Khaleghi Bizaki, ${ }^{1}$ Morteza Khaleghi Hojaghan, \\ and Seyyed Mohammad Razavizadeh ${ }^{3}$ \\ ${ }^{1}$ Malek Ashtar University of Technology, Tehran, Iran \\ ${ }^{2}$ Tabriz University, Tabriz, Iran \\ ${ }^{3}$ Iran University of Science and Technology (IUST), Tehran, Iran \\ Correspondence should be addressed to Hossein Khaleghi Bizaki; bizaki@yahoo.com
}

Received 28 January 2013; Revised 15 April 2013; Accepted 27 April 2013

Academic Editor: Kai-Kit Wong

Copyright @ 2013 Hossein Khaleghi Bizaki et al. This is an open access article distributed under the Creative Commons Attribution License, which permits unrestricted use, distribution, and reproduction in any medium, provided the original work is properly cited.

\begin{abstract}
This paper concentrates on the designing of a robust Tomlinson-Harashima Precoder (THP) over multiple-input multiple-output (MIMO) channels in wireless communication systems with assumption of imperfect channel state information (CSI) at the transmitter side. With the assumption that the covariance matrix of channel estimation error is available at the transmitter side, we design a THP that presents robustness against channel uncertainties. In the proposed robust THP, the transmit power is further minimized by using the Tilted constellation concept. This power minimization reduces the interchannel Interference (ICI) between subchannels and, furthermore, recovers some part of the THP's power loss. The bit error rate (BER) of the proposed system is further improved by using a power loading technique. Finally, the simulation results compare the performance of our proposed robust THP with a conventional MIMO-THP.
\end{abstract}

\section{Introduction}

Recently, due to the impressive capacity of multiple-input multiple-output (MIMO) channel, many companies and researches have been attracted toward designing transceivers for MIMO systems and especially robust transceivers in the case of channel uncertainties. It is well known that inter channel interference (ICI) is one of the important problems of the MIMO systems. It is possible to preeliminate the ICI at the transmitter side by using precoding techniques and consequently reduce transmission power. Due to channel estimation errors, channel variations during time, and channel feedback errors, the assumption of perfect CSI at the transmitter side is not always true and hence the CSI at the transmitter side has uncertainties [1]. Due to these uncertainties, the ICI will be increased and consequently leads to BER growth. Linear precoder in [2] is designed for imperfect CSI, but it could only slightly reduce uncertainties effects by using statistical information of the channel. Nonlinear TomlinsonHarashima Precoder (THP) in [3] has been considered for imperfect CSI assumption, and we combine it with the tilted constellation method in our work.

In this paper, we propose a design of a robust MIMOTHP that can reduce uncertainties effects more than the proposed methods in $[2,3]$ and subsequently can further reduce BER. Similar to [3], we utilize second-order statistics of the uncertainties of the MIMO channels at the transmitter side. Moreover, by using tilted constellation concept in [4], we can significantly reduce transmit power. It should be noted that transmit power reduction can decrease ICI between subchannels and in addition recover some parts of the THP's power loss. This ICI reduction between subchannels is one of the main challenges in the designing of our precoder.

Tilted constellation method is introduced in [4] for SISO channel. Based on this idea, we have extended it for the MIMO channel. Here, the power reduction is done by tilting or rotating the ordinary constellation through a proper angle. The desired angle is one that minimizes the transmit power and is selected from a set of possible angles. The results show that the power reduction will be greater at high SNRs values. 


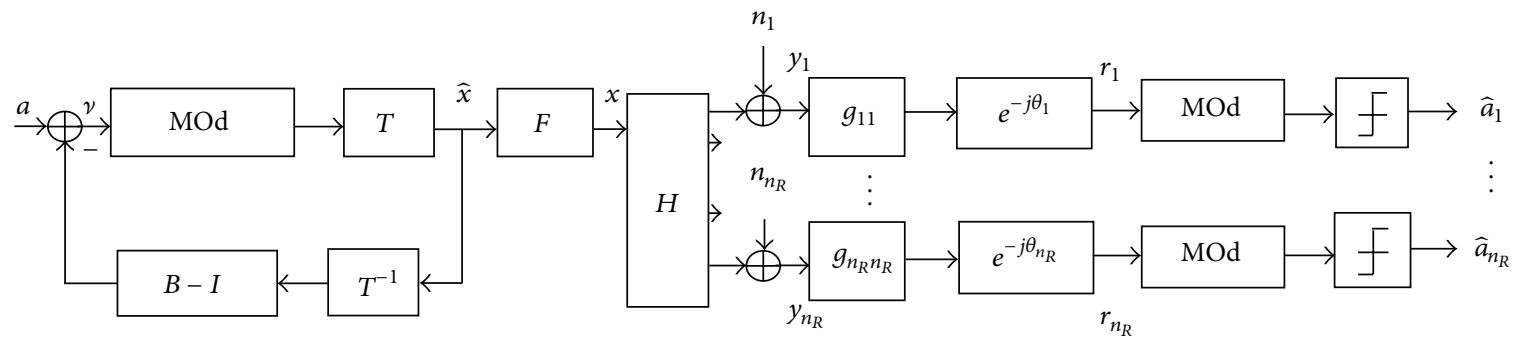

FIgURE 1: The proposed tilted MIMO-THP in decentralized scenario.

To do the tilted constellation method, we use different angles for tilting the constellation in each antenna. Then, we find a set of proper angles, which are selected in a manner to minimize the transmit power in all antennas. The transmit power reduction can reduce the transmission block power, lower than the power of modulated symbols in each block. Finally, we can send proper angles to the receiver within the main data frame. We will show that the proposed method outperforms the conventional MIMO-THP in most SNRs and also in all symbol block lengths.

We can adjust transmit power of each antenna when the CSI (perfect/imperfect) is available at the transmitter side which is known as power loading [5]. It should be noted that in the MIMO systems some of the corresponding parallel subchannels (corresponding to each antenna) might have very low channel condition or might be useless for sometimes [5]. In this situation, the transmitter can adjust transmit power of each antenna by redistributing the available transmit power to get a better average error rate [5]. Moreover, by utilizing power loading, we improve the BER performance of the tilted MIMO-THP. We also study the complexity of our proposed method with respect to some previous methods.

This paper is organized as follows. In Section 2, the system model of the Tilted MIMO-THP in imperfect CSI scenario is introduced. In Section 3, the proposed robust tilted MIMOTHP is described for imperfect CSI. Section 4 presents the power loading technique to improve the performance of the robust precoder. In Section 5, the simulation results are given to show the performance of the proposed method and its comparison with the conventional MIMO-THP. Finally, conclusions are drawn in Section 6.

\section{System Model}

In this paper, an MIMO communication system with $n_{T}$ transmit antenna at the base station and $n_{R}$ users at the receivers side, each equipped with one receive antenna, are considered. The block diagram of the considered system is shown in Figure 1.

The $n_{T}$ input data symbols and the transmitted signals are denoted by vector $\mathbf{a}$ and vector $\mathbf{x}$, respectively. Both vectors are of size $n_{T} \times 1$. The channel matrix is modeled as $\mathbf{H}=$ $\mathbf{H}_{\mathbf{0}}+\Delta \mathbf{H}$, where $\mathbf{H}_{\mathbf{0}}$ is the $n_{R} \times n_{T}$ estimated channel matrix whose elements are independent and identically distributed (i.i.d.) zero mean complex Gaussian random variables with variance $1-\rho^{2}$ (i.e, $\mathbf{H}_{\mathbf{0}} \sim \mathrm{CN}\left(0,1-\rho^{2}\right)$ ), and $\Delta \mathbf{H}$ is an $n_{R} \times n_{T}$ channel uncertainties matrix whose elements are i.i.d. zero mean complex Gaussian random variables with variance $\rho^{2}$ (i.e, $\left.\Delta \mathbf{H} \sim \mathrm{CN}\left(0, \rho^{2}\right)\right)$. Thus, $\mathbf{H}$ can be modeled as $\mathbf{H} \sim \mathrm{CN}(0,1)$. The matrix $\mathbf{T}$ is the proper rotation angles matrix which is named as tilting matrix, to minimize the transmission power. The received signal $\mathbf{y}$ can be denoted as follows:

$$
\mathbf{y}=\mathbf{H x}+\mathbf{n}
$$

where $\mathbf{n}$ is the noise vector whose elements are assumed to be i.i.d. complex Gaussian random variables with zero mean and variance $\sigma^{2}$ (i.e. $\mathbf{n} \sim \mathrm{CN}\left(0, \sigma^{2} \mathbf{I}_{\mathbf{R}}\right)$ ).

As we see in Figure 1, the $n_{T}$ dimensional vector a corresponds to input data, and $n_{T}$ dimensional vector $\mathbf{x}$ represents transmitted signals. The feedback matrix $\mathbf{B}$ is added to preeliminate the interference from previous users, and the tilting matrix $\mathbf{T}$ is added to reduce the transmit power. Finally, in order to keep the power of transmitted symbols within the original constellation boundary, we employ modulo operator at the forward loop. The boundary region of the constellation is related to the order of modulation constellation, which for rectangular $M$-ary QAM modulation is $t=2 \sqrt{M}$. Using tilted constellation, the signal constellation is further rotated by a set of appropriate angles, represented by the diagonal elements of the Tilting matrix T, to reduce the ICI and power loss of THP.

The resultant signal $\widehat{\mathbf{x}}$ is then passed through a unitary feed-forward filter $\mathbf{F}$, to eliminate the residual interference. Finally, the precoded signal is sent through the MIMO channel. All processes to eliminate the interference are performed at the transmitter side; hence, receivers at the user side are left with some simple operations including power scaling (i.e., elements of the diagonal matrix $\mathbf{G}$ ), de-modulo operation, constellation tilting with reverse angle, and a single user detection.

By using the second-order statistics of the uncertainties of the MIMO channels at the transmitter side, we design a THP that presents robustness against channel uncertainties. We can describe the difference between impressive vector $\mathbf{v}$ and input vector to the decision block as follows [3]:

$$
\mathbf{e}=\mathbf{r}-\mathbf{v}=\left[\mathbf{T}^{-1} \mathbf{G}\left(\mathbf{H}_{\mathbf{0}}+\Delta \mathbf{H}\right) \mathbf{F}-\mathbf{B}\right] \widehat{\mathbf{x}}+\widetilde{\mathbf{n}} .
$$

MMSE solution should minimize the signal error as:

$$
\begin{array}{r}
\arg \min _{\mathbf{B}, \mathbf{F}, \mathbf{G}} E\left\|\left[\left[\mathbf{T}^{-\mathbf{1}} \mathbf{G}\left(\mathbf{H}_{\mathbf{0}}+\mathbf{\Delta} \mathbf{H}\right) \mathbf{F}-\mathbf{B}\right] \widehat{\mathbf{x}}+\widetilde{\mathbf{n}}\right]\right\|^{2} \\
\text { so that } E\|\widehat{\mathbf{x}}\|^{2} \leq P_{T},
\end{array}
$$


where $P_{T}$ is the total transmitted power. Since, we assume the matrix $\mathbf{F}$ is unitary thus, the power constraint is guaranteed.

Instead of analysis of (3) we can use orthogonality property as follow [3]:

$$
E\left[\mathbf{e r}^{H}\right]=0
$$

Hence from (4) we have the following:

$$
\mathbf{G} \Phi_{\mathbf{r r}}=\mathbf{B} \Phi_{\mathbf{x r}}
$$

We can calculate $\Phi_{\mathbf{r r}}$ and $\Phi_{\mathbf{x r}}$ using (1) as follows:

$$
\begin{gathered}
\Phi_{\mathbf{r r}}=E\left[\mathbf{r r}^{H}\right]=\boldsymbol{\sigma}_{\mathbf{x}}^{2}\left(\mathbf{H}_{\mathbf{0}} \mathbf{H}_{\mathbf{0}}{ }^{H}+\boldsymbol{\zeta} \mathbf{I}+\mathbf{C}_{\Delta \mathbf{H}}\right) \\
\Phi_{\mathbf{x r}}=E\left[\mathbf{x r}^{H}\right]=\boldsymbol{\sigma}_{\mathbf{x}}^{2}\left(\mathbf{F}^{H} \mathbf{H}_{\mathbf{0}}{ }^{H}\right)
\end{gathered}
$$

By substituting (6) into (5), and after some manipulations, we have the following:

$$
\mathbf{F}^{H}=\mathbf{B}^{-1} \mathbf{G}\left(\mathbf{H}_{\mathbf{0}} \mathbf{H}_{\mathbf{0}}{ }^{H}+\zeta \mathbf{I}+\mathbf{C}_{\Delta \mathbf{H}}\right) \mathbf{H}_{\mathbf{0}}{ }^{-H} .
$$

Since $\mathbf{F}$ is unitary, we have the following:

$$
\mathbf{R R}^{H}=\left(\mathbf{H}_{\mathbf{0}} \mathbf{H}_{\mathbf{0}}{ }^{H}+\zeta \mathbf{I}+\mathbf{C}_{\Delta \mathbf{H}}\right) \mathbf{H}_{\mathbf{0}}{ }^{-H} \mathbf{H}_{\mathbf{0}}{ }^{-1}\left(\mathbf{H}_{\mathbf{0}} \mathbf{H}_{\mathbf{0}}{ }^{H}+\zeta \mathbf{I}+\mathbf{C}_{\Delta \mathbf{H}}\right)
$$

where $\mathbf{R}=\mathbf{G}^{-1} \mathbf{B}$. Matrix $\mathbf{R}$ can be obtained by doing the Cholesky factorization of (8). We can calculate matrixes $\mathbf{G}$, $\mathbf{B}$, and $\mathbf{F}$ as follows:

$$
\begin{gathered}
\mathbf{G}=\operatorname{diag}\left(\frac{\mathbf{1}}{\mathbf{r}_{11}}, \ldots, \frac{\mathbf{1}}{\mathbf{r}_{n_{T} n_{T}}}\right), \\
\mathbf{B}=\mathbf{G}\left(\mathbf{H}_{\mathbf{0}} \mathbf{H}_{\mathbf{0}}{ }^{H}+\zeta \mathbf{I}+\mathbf{C}_{\Delta \mathbf{H}}\right) \mathbf{H}_{\mathbf{0}}{ }^{-H}=\mathbf{G R}, \\
\mathbf{F}=\mathbf{H}_{\mathbf{0}}{ }^{-1}\left(\mathbf{H}_{\mathbf{0}} \mathbf{H}_{\mathbf{0}}{ }^{H}+\zeta \mathbf{I}+\mathbf{C}_{\Delta \mathbf{H}}\right) \mathbf{R}^{-H} .
\end{gathered}
$$

Substituting G, B, and F into (9) the error covariance matrix is equal to the following:

$$
\Phi_{\mathbf{e e}}=E\left[\mathbf{e} \mathbf{e}^{H}\right]=\boldsymbol{\sigma}_{\mathbf{x}}^{2} \mathbf{G}\left(\zeta^{2} \mathbf{H}_{\mathbf{0}}{ }^{-H} \mathbf{H}_{\mathbf{0}}{ }^{-1}+\zeta \mathbf{I}+\mathbf{C}_{\Delta \mathbf{H}}\right) \mathbf{G}^{H},
$$

where the scalar $\zeta$ represents the $1 / \mathrm{SNR}=\sigma_{n}^{2} / \sigma_{x}^{2}$.

It can be seen in (10) that, while the channel has uncertainty, the error covariance matrix has an additional term of $\boldsymbol{\sigma}_{\mathbf{x}}^{2} \mathbf{G C}_{\Delta \mathbf{H}} \mathbf{G}^{H}$ with respect to its counterpart in perfect CSI case.

In this paper, we assume the previous relations and matrixes as the conventional robust MIMO-THP, that is, without using tilted constellations.

\section{Robust Tilted MIMO-THP}

As mentioned before, in the general model of Figure 1, each diagonal element of the Tilting matrix $\mathbf{T}$ determines the rotation angle of its corresponding antenna signal.

In conventional THP, the main constellation is only extended by the modulo operator on its boundary regions.
However, in the tilted-THP, the signal constellation is further rotated by a set of appropriate angles. To do this, first a block of symbols with length $n_{T} \times N$ is divided to $n_{T}$ groups each of length $N$. Then, for every group of symbols which is transmitted from each antenna, the transmitter chooses an appropriate angle from a set of possible angles in such a way that the transmitted power is minimized based on some predefined criteria. From [4], the ordinary constellation is tilted by $Q$ possible angles as $\theta_{q} ; q=1, \ldots, Q$, then the optimal angle $\theta_{q}^{*}$ is selected by the following equation:

$$
q^{*}=\arg \min _{q=1, \ldots, Q}\left\|\operatorname{Mod}\left[\mathbf{a}-\mathbf{i} \mathbf{e}^{-j \theta_{q}}\right]\right\|,
$$

where $\mathbf{a}$ is the group of symbols in each transmit antenna, and $\mathbf{i}$ illustrates the interference sequence at each antenna due to the previous antennas and is calculated similar to the conventional THP. The difference between the conventional (i.e. un-tilted) and tilted THP is in rotating of the transmitted signal of the previous antennas by their optimal angles. Transmitted power and resulting optimal angle of each antenna can be calculated as follows [4]:

$$
\begin{gathered}
P_{\text {Tilted }}(Q)=E\left\{\min _{q=1, \ldots, Q} \mathbf{P}_{q}\right\} \\
\mathbf{P}_{q}=\frac{1}{N} \sum_{i=1}^{N}\left\|\operatorname{Mod}\left[\mathbf{a}-\mathbf{i} e^{-j \theta_{q}}\right]\right\|^{2} .
\end{gathered}
$$

The vector $\mathbf{a}_{1}$, which is transmitted from the first transmit antenna, is not affected by any interference signal, that is why we transmit $\mathbf{a}_{1}$ without any interference. The optimal tilted angle $\theta_{1}$ is resulted by minimizing the transmitted power of the first antenna; that is:

$$
\mathbf{x}_{\mathbf{1}}=\mathbf{a}_{1} e^{j \theta_{1}} .
$$

For $\mathbf{a}_{2}$, which is transmitted from the second antenna, the optimal transmit angle is achieved by computing the minimum transmitted power similar to the $\mathbf{a}_{1}$, but, here, there is an interference due to the $\mathbf{a}_{1}$, that is, $\mathbf{b}_{21}$, and hence we have the following:

$$
\mathbf{x}_{2}=\left(\operatorname{Mod}\left(\mathbf{a}_{2}-\mathbf{b}_{21} \mathbf{x}_{1} e^{-j \theta_{2}}\right)\right) \mathbf{e}^{j \theta_{2}} .
$$

Similarly, for the $k$ th antenna, we have the following:

$$
\mathbf{x}_{k}=\left(\operatorname{Mod}\left(\mathbf{a}_{k}-\sum_{j=1}^{k-1} \mathbf{b}_{k j} \mathbf{x}_{j} e^{-j \theta_{k}}\right)\right) e^{j \theta_{k}},
$$

where $\theta_{k}$ is the optimal tilted angle for the $k$ th antenna. At the receiver side, the received signal for the $k$ th antenna can be shown as follows:

$$
\mathbf{r}_{k}=\left(\mathbf{b}_{k} \mathbf{x}+\mathbf{n}_{k}\right) e^{-j \theta_{k}},
$$

where $\mathbf{b}_{k}$ denotes the $k$ th row of the matrix $\mathbf{B}=\left[\mathbf{b}_{1}, \ldots\right.$, $\left.\mathbf{b}_{k, \ldots}\right]^{\mathrm{T}}$. By using (15), (16) can be written as

$$
\mathbf{r}_{k}=\left(\mathbf{x}_{k}+\sum_{j=1}^{k-1} \mathbf{b}_{k j} \mathbf{x}_{j}+\mathbf{n}_{k}\right) e^{-j \theta_{k}} .
$$


Since $b_{k k}=1$, after modulo operator we obtain the following:

$$
\begin{aligned}
\widehat{\mathbf{a}}_{k} & =\operatorname{Mod}\left(\mathbf{r}_{k}\right) \\
& =\operatorname{Mod}\left(\left(\mathbf{a}_{k} e^{j \theta_{k}}-\sum_{j=1}^{k-1} \mathbf{b}_{k j} \mathbf{x}_{j}+\sum_{j=1}^{k-1} \mathbf{b}_{k j} \mathbf{x}_{j}+\mathbf{n}_{k}\right) e^{-j \theta_{k}}\right) \\
& =\operatorname{Mod}\left(\left(\mathbf{a}_{k} e^{j \theta_{k}}+\mathbf{n}_{k}\right) e^{-j \theta_{k}}\right)=\operatorname{Mod}\left(\mathbf{a}_{k}+\mathbf{n}_{k} e^{-j \theta_{k}}\right) .
\end{aligned}
$$

As shown in [3], in the tilted constellation case, the matrix $\mathbf{B}$ is similar to the untilted or regular MIMO-THP case. Its difference is only in rotation of the phase of the noise. Since the noise has circularly symmetric complex Gaussian distribution, for the conventional case, all equations and results for the decomposition of matrices in the tilted constellation case are the same.

If we use the tilted constellation for detecting the original symbols directly, the performance will be degraded due to rotation and reduction of minimum distance between symbols. Hence, we should send the optimal angles to the receiver to detect the desired symbols. Upon receipt, first, the symbols are reversely rotated at each antenna and then are detected in a way similar to the conventional THP.

\section{Power Loading}

The SER of MIMO-THP in imperfect CSI can be approximated as follows [6]:

$$
\mathrm{SER} \approx \sum_{k=1}^{K}\left(1-\frac{1}{\sqrt{M_{k}}}\right) Q\left(\sqrt{\frac{3}{M_{k}-1} \frac{\left(P_{\text {Tilted }}\right)_{k}}{\sigma_{e}^{2}}}\right),
$$

where $\left(P_{\text {Tilted }}\right)_{k}$ is the transmitted power of $k$ th transmit antenna and $\sigma_{e}^{2}$ is noise power [3]:

$$
\sigma_{e}^{2}=\left[\Phi_{\mathbf{e e}}\right]_{k k}=\frac{1}{\left|\mathbf{r}_{k k}\right|^{2}}\left(\sigma_{n}^{2}+\sum_{j=1}^{K}\left(P_{\text {Tilted }}\right)_{j}\left|\delta_{k j}\right|^{2}+\beta_{k}\right) .
$$

Here, $\delta_{i j}=[\Delta \mathbf{H}]_{i j}, \beta_{k}=\sigma_{n}^{4} \sum_{j=1}^{K}\left|h_{k j}\right|^{2} /\left(P_{\text {Tilted }}\right)_{j}$, and $h_{i j}=\left[\mathbf{H}^{-H} \mathbf{H}^{-1}\right]_{i j}$. With the assumption of small error, that is, $\alpha\left(P_{\text {Tilted }}\right)_{j} \leq \sigma_{n}^{2}$; for all $j[3]$, we can approximate the $\sigma_{e}^{2}$ value as follows:

$$
\sigma_{e}^{2}=\frac{1}{\left|\mathbf{r}_{k k}\right|^{2}}\left(\sigma_{n}^{2}+\alpha\left(P_{\text {Tilted }}\right)_{T}+\beta_{k}\right),
$$

where $\left(P_{\text {Tilted }}\right)_{T}$ is the total tilted power. At the above equation, we assumed the worst-case (i.e., $\alpha=\max _{\mathbf{B}, \mathbf{F}, \mathbf{G}}$ $\left|\delta_{i j}\right|^{2}$; for all $\left.i, j\right)$. We distribute the power to minimize SER in imperfect CSI by using the Lagrange method, so that it can be written as follows:

$$
\begin{aligned}
L= & \sum_{k=1}^{K}\left(1-\frac{1}{\sqrt{M_{k}}}\right) Q\left(\sqrt{\left(P_{\text {Tilted }}\right)_{k} \beta_{k}}\right) \\
& -\lambda\left(K-\sum_{k=1}^{K}\left(P_{\text {Tilted }}\right)_{k}\right) .
\end{aligned}
$$

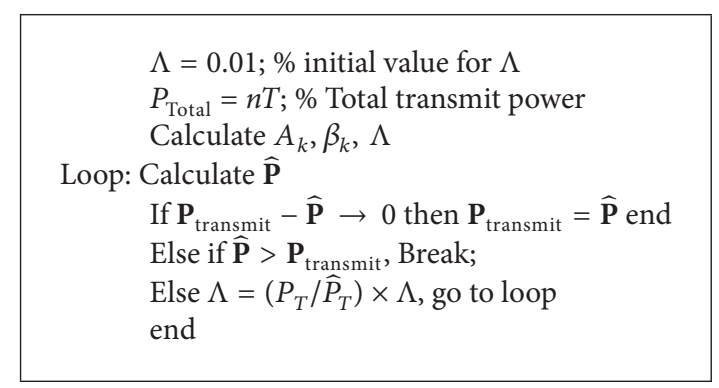

Algorithm 1: The proposed power loading algorithm.

Hence, as in [3], we have the following:

$$
\beta_{k}=\frac{3}{M_{k}-1} \frac{1}{\sigma_{n}^{2}+\alpha\left(P_{\text {Tilted }}\right)_{T}+\beta_{k}} .
$$

Unfortunately, we did not find any closed form solution for (23), so we should use some suboptimal solution as in [3].

By Solving $\partial L / \partial\left(P_{\text {Tilted }}\right)_{k}=0$ for $\left(P_{\text {Tilted }}\right)_{k}$, we have the following:

$$
\left(P_{\text {Tilted }}\right)_{k}=\frac{1}{\beta_{k}} W\left(\frac{\beta_{k}}{A_{k}} \Lambda\right),
$$

where $A_{k}=\left(M_{k} /\left(\sqrt{M_{k}}-1\right)^{2}\right)\left(1 / \beta_{k}\right), \beta_{k}=\left(3 /\left(M_{k}-1\right)\right)$ $\left(\left|r_{k k}\right|^{2} /\left(\sigma_{n}^{2}+\alpha\left(P_{\text {Tilted }}\right)_{T}+\beta\right)\right), \Lambda=$ cte, and $W(x)$ is real Lambert function [3].

Thus, the power vector $\mathbf{P}=\left[\left(P_{\text {tilted }}\right)_{1}, \ldots,\left(P_{\text {tilted }}\right)_{n_{T}}\right]$ can be obtained by using the following unique repeated solution [3].

(I) A small positive value $\Lambda$ is chosen so that

$$
\sum_{k=1}^{K} \frac{1}{A_{k}} \leq P_{T}
$$

(II) The value $\widehat{P}_{T}$ is calculated as follows:

$$
\widehat{P}_{T}=\sum_{k=1}^{K} \frac{1}{\beta_{k}} W\left(\frac{\beta_{k}}{A_{k}} \Lambda\right) .
$$

(III) If $\widehat{P}_{T}$ is not close enough to $P_{T}, \Lambda$ multiply $P_{T} / \widehat{P}_{T}$ and be returned to the stage II.

(IV) Based on (24), vector $\mathbf{P}$ is calculated.

This algorithm is present as pseudocode in Algorithm 1.

Now, we discuss the complexity of the proposed method. We realize that the proposed method adds some parts to the conventional THP method. To compare the complexities of the proposed method and the conventional THP, we only consider those parts that are changed in our method. For example, for the case of $n_{R}=n_{T}=4$, the complexity of the conventional THP is $15 n$ at the transmitter side and is $8 n$ at the receiver side while for the proposed Tilted-THP method this complexity is $25 n$ at the transmitter side and is 


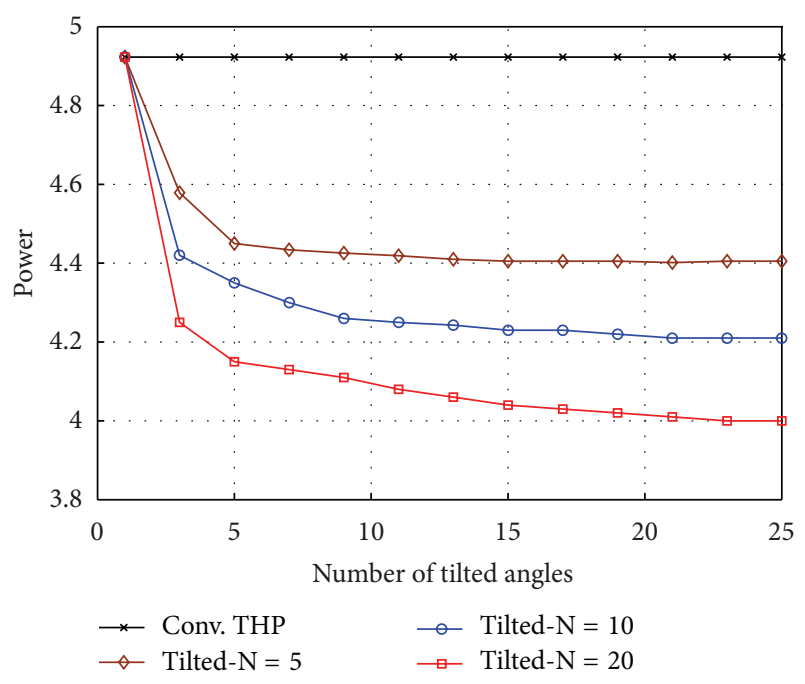

FIGURE 2: Transmitted power versus the number of tilted angles in different block length $N=5,10,20\left(\mathrm{SNR}=10 \mathrm{~dB}, n_{T}=n_{R}=4\right.$, 4QAM).

$12 n$ at the receiver side, where $n=N / n_{T}$ is the length of the input symbol to each antenna. As it can be seen, the increased complexity is suitable. On the other hand, as we will show in the next section in simulation results, the performance gain that we attain by the proposed method is more considerable with respect to the above complexity increasing.

\section{Simulation Results}

To evaluate the performance of our proposed method, we use some simulations. To do this we assume an MIMO-THP system with 4 transmit and 4 receive antennas with 4-QAM modulation. We assume that the channel estimation error covariance $\rho=0.1$ and 0.05 . In our figures, the labels "conv," "Rob," and "P.L." are corresponding to conventional, robust, and power loading methods, respectively.

Figure 2 shows the transmitted power of the MMSE-THP system versus the number of tilted angles. In this figure, the SNR is assumed to be $10 \mathrm{~dB}$. It can be seen that by increasing the number of available tilted angles, the transmitted power reduces until it converges to a constant floor. Also, it can be seen that by decreasing the block length, the power reduction will be more and the power converges faster to its floor. The reason of this floor is that we assumed that the matrix $\mathbf{F}$ is unitary. As can be seen, for example, the transmitted power for the conventional MIMO-MMSE-THP for $Q=25$ is 4.91 but, for the Tilted constellation and with the same parameters, the transmitted power decreases to 4 and 4.24 when the block length increases to 5 and 10, respectively.

Figure 3 shows BER of the MMSE and robust-tiltedMMSE precoders versus the Eb/No for different block lengths and for $\rho=0.05$ with and without the power loading. As it is seen in this figure, with the Robust-Tilted THP, the BER decreases compared to the conventional one. This BER reduction is more when we use the power loading. Moreover, because of more power reduction in smaller block lengths,

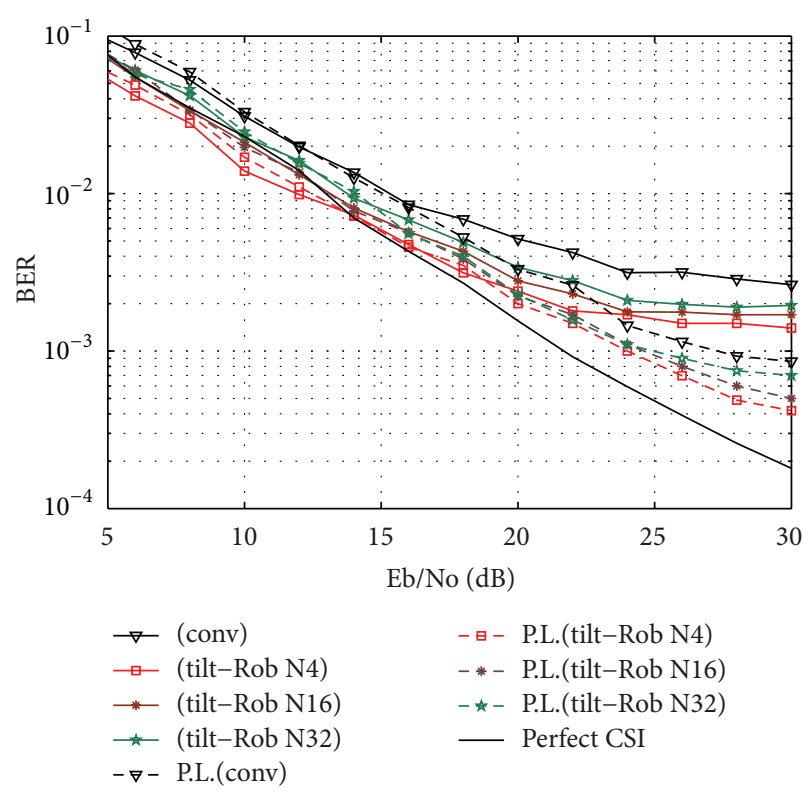

FIGURE 3: BER versus SNR and several block length in MIMOMMSE-THP with and without power loading $(\rho=0.05)$.

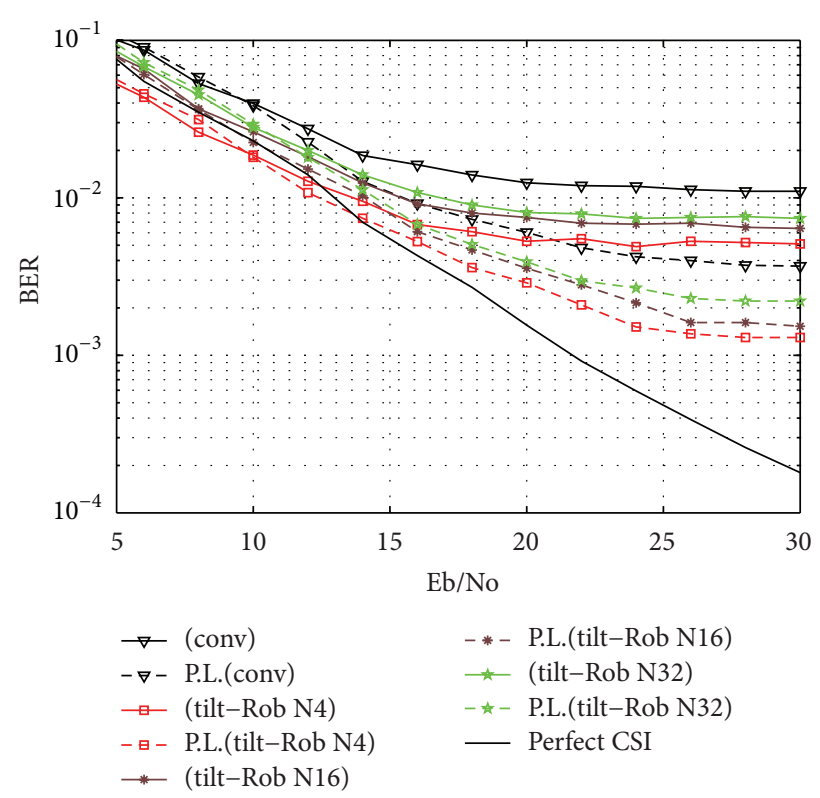

FIGURE 4: BER versus SNR and several block length in MIMOMMSE-THP with and without power loading $(\rho=0.1)$.

the BER reduction will be more in smaller block length. For example, for SNR $=30 \mathrm{~dB}$ and $N=4$ in the conventional MIMO-MMSE-THP, we have BER $=0.0028$ while in the robust Tilted constellation MIMO-MMSE-THP for with and without power loading we have $\mathrm{BER}=0.0015$ and $\mathrm{BER}=$ 0.0004 , respectively.

Figure 4 shows BER of the MMSE and Robust-TiltedMMSE precoders versus the $\mathrm{Eb} / \mathrm{No}$ for different block lengths and for $\rho=0.1$ with and without the power loading. As it is seen in this figure, with the Robust-Tilted constellation, BER 
decreased compared to the conventional MIMO-THP which is similar to Figure 3. Again, BER reduction is more when we use the power loading. Due to more power reduction of Tilted constellation for shorter block length, the resultant BER has reduced more for shorter block lengths. More channel uncertainties with respect to Figure 3 lead to BER incensement. For example, at $\mathrm{SNR}=30 \mathrm{~dB}$ in the conventional MIMO-MMSETHP, BER $=0.011$ while in the robust Tilted constellation MIMO-MMSE-THP for $N=4$ with and without power loading BER $=0.005$ and $\mathrm{BER}=0.0012$, respectively.

\section{Conclusion}

In this paper, we proposed a robust-tilted-MIMO-THP scheme for MIMO channels based on MMSE criterion with the assumption that the imperfect CSI is available at the transmitter side. We used second-order terms of the uncertainties of the MIMO channels at the transmitter to design a robust precoder. In the proposed robust method, we minimized the transmit power by using the Tilted constellation. The power minimization will result in ICI reduction between subchannels and in addition recovering some of the THP's power loss. Consequently, this ICI reduction and power recovering result led to BER improvement, especially at high SNRs. The transmission power is more reduced by utilizing more Tilted angles and smaller symbol block length. Also, we demonstrated that the achieved performance gain by using the proposed method is more considerable with respect to its higher complexity. Finally, we showed that we can improve our precoder performance further, by utilizing power loading.

\section{References}

[1] X. Geng, L. Jiang, and C. He, "Robust design for generalized vector precoding by minimizing mse with imperfect channel state information," Chinese Journal of Electronics, vol. 19, no. 3 , pp. 503-506, 2010.

[2] N. Khaled, G. Leus, C. Desset, and H. Man, "A robust joint linear precoder and decoder MMSE design for slowly time-varying MIMO channels," in Proceedings of the IEEE International Conference on Acoustics, Speech, and Signal Processing (ICASSP '04), pp. iv-485-iv-488, May 2004.

[3] H. K. Bizaki and A. Falahati, "Tomlinson-Harashima precoding with imperfect channel state information," IET Communications, vol. 2, no. 1, pp. 151-158, 2008.

[4] J. Kang, H. Ku, D. S. Kwon, and C. Lee, "Tomlinson-harashima precoder with tilted constellation for reducing transmission power," IEEE Transactions on Wireless Communications, vol. 8, no. 7, pp. 3658-3667, 2009.

[5] H. K. Bizaki and A. Falahati, "Power loading by minimizing the average symbol error rate on MIMO THP systems," in Proceedings of the 9th International Conference on Advanced Communication Technology (ICACT '07), pp. 1323-1326, February 2007.

[6] J. G. Proakis and M. Salehi, Digital Communications, McGrawHill, 5th edition, 2008. 


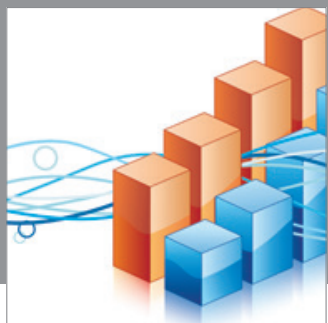

Advances in

Operations Research

mansans

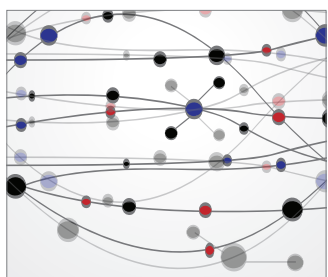

The Scientific World Journal
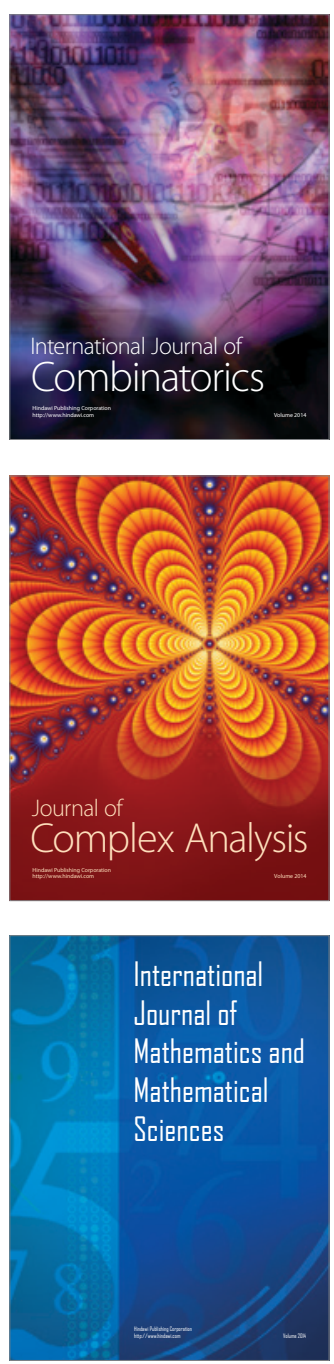
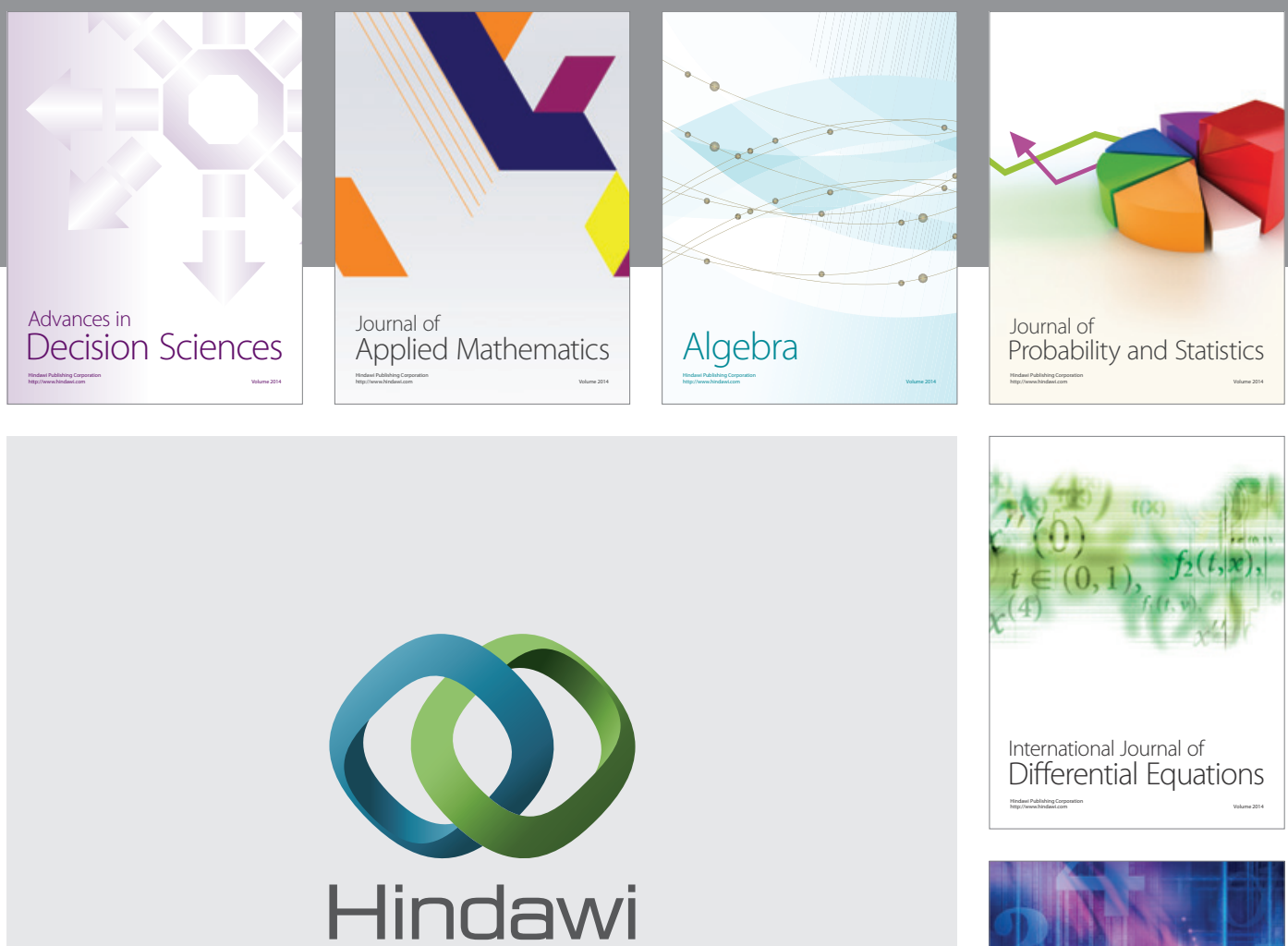

Submit your manuscripts at http://www.hindawi.com
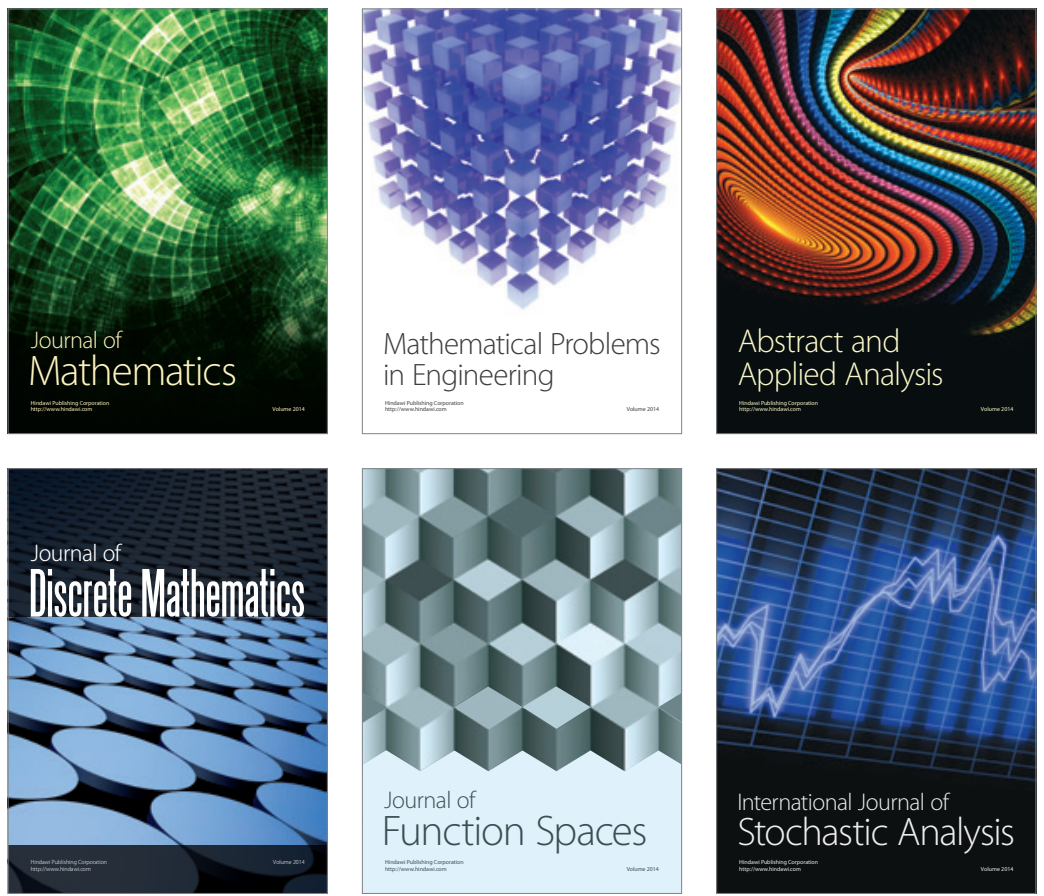

Journal of

Function Spaces

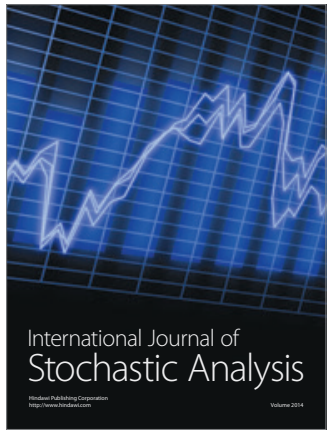

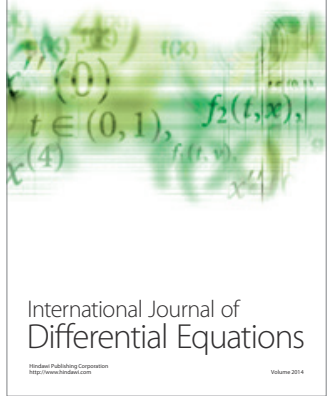
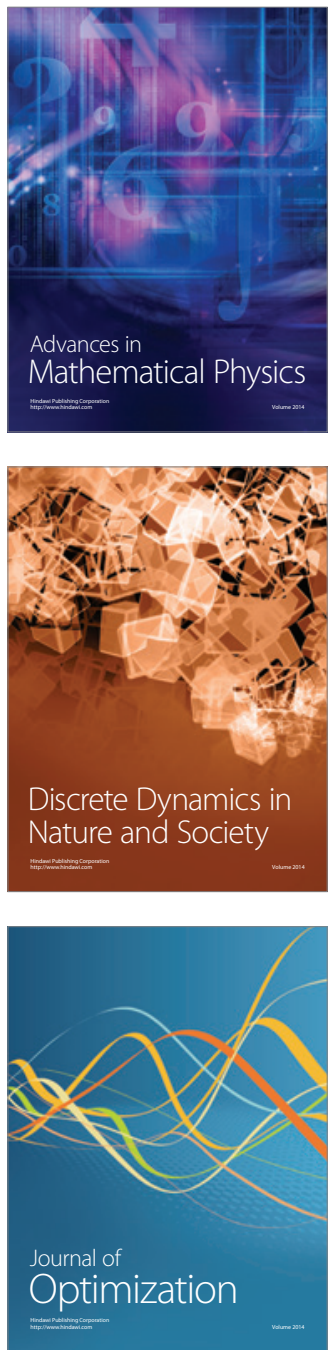